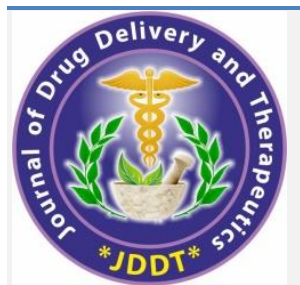

Open

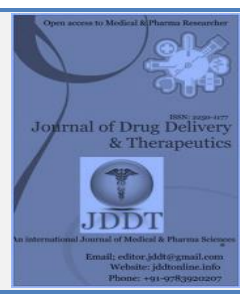

Research Article

\title{
The Role of Clinical Pharmacist in Enhancement of Medication Adherence and Quality of Life in Bronchial Asthma and Chronic Obstructive Pulmonary Disease
}

\author{
Aswathi Gangadharan ${ }^{1}$, Diya Sheela Thomas ${ }^{1}$, Harshida ${ }^{1}$, Cyril Tom ${ }^{1 *}$, Prabhakar Adake ${ }^{2}$ \\ ${ }^{1}$ Department of Pharmacy Practice, Shree Devi College of Pharmacy, Mangalore, Karnataka, India \\ ${ }^{2}$ Department of Pharmacology, Yenepoya Medical College Hospital, Derlakatte, Mangalore, Karnataka, India
}

Article Info:

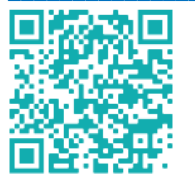

\section{Article History:}

Received 09 June 2021

Reviewed 20 July 2021

Accepted 27 July 2021

Published 15 August 2021

\section{Cite this article as:}

Gangadharan A, Thomas DS, Harshida, Tom C, Adake P, The Role of Clinical Pharmacist in

Enhancement of Medication Adherence and Quality of Life in Bronchial Asthma and Chronic

Obstructive Pulmonary Disease, Journal of Drug Delivery and Therapeutics. 2021; 11(4-S):48-53

DOI: http://dx.doi.org/10.22270/jddt.v11i4-S.4952

*Address for Correspondence:

Cyril Tom, Department of Pharmacy Practice, Shree Devi College of Pharmacy, Mangalore, Karnataka, India

\section{Abstract}

Asthma and Chronic Obstructive Pulmonary Disease (COPD) are two of the leading causes of morbidity, mortality and economic burden worldwide. The burden of chronic respiratory disease has major adverse effects on the quality of life and ability of affected individuals. A variety of effective treatment options exist for patients with Asthma and COPD but long-term adherence to medications is required for treatment success. Aim of the study was to assess the adherence to medication and quality of life in Asthma and COPD patients using questionnaires, and to enhance adherence and quality of life using the strategies of counselling and education. The observational study was conducted for a period of 6 months, assessed medication adherence by Morisky self-reported questionnaires and quality of life by St. George's Respiratory questionnaire (SGRQ) and 36 item short form health survey (SF-36). The study shows there was statistically significant improvement in medication adherence score $(p<0.05)$ and quality of life $(p<0.05)$ after pharmacist's counselling regarding disease, medication and lifestyle. Comparison between various demographic factors like age, gender, co-morbidities, smoking index showed equal impact of pharmacist's counselling on improvement of overall medication adherence and quality of life in Asthma and COPD patients. Overall medication adherence and quality of life improvement was found to be statistically significant in Asthma and COPD patients after counselling by clinical pharmacists. Clinical pharmacists must be considered as an integral element of healthcare system. Patient counselling is effective to resolve the problems associated with medication non-adherence and quality of life.

Keywords: Asthma, COPD, Medication adherence, Quality of life

\section{INTRODUCTION}

Medication adherence is one of the most important factors that determine therapeutic outcome especially in patients suffering from chronic illness. Low medication adherence will undermine the benefits of the medical care.

Patient adherence can be defined as an extent to which patient behaviour coincides with health-related advice and ability of patient to take medications as prescribed. Adherence to the medication has reported to be low among patients with COPD and Asthma. The major reasons for medication non-compliance were felt better and stopped, high cost of medications, forgetfulness, fear of side effects, non-beneficial treatment. Poor compliance with prescribed therapy leads to increased morbidity and mortality. ${ }^{1}$

Quality of life is defined by World Health Organization (WHO) as a broad and complex concept of individual about their physical health, mental health and social relationship. Chronic illness of COPD and Asthma are frequent in general population. These diseases are characterised by a narrowing of bronchi associated with chronic inflammation. However, there are some differences between Asthma and COPD in terms of onset age, casual factors, clinical aspects and impact on daily life. However, the relationship between pulmonary obstruction, dyspnoea and impact of the disease on the daily life of the patient is not necessarily linear.

Questionnaires do exist and have been used for decades as they can gather data in a standardised form, about the patient's perception of the status of disease and treatment. This information reported directly by the patient without other people's interpretation about his/her well- being, behaviour and feelings in regard to state of health. ${ }^{2}$

\section{MATERIAL AND METHODS}

The study was approved by the Yenepoya University Ethics Committee, Deralakatte, Mangalore. In this study, 69 cases were collected in which the role of clinical pharmacist in enhancement of medication adherence and quality of life in COPD and Asthma patients were studied. This Prospective Observational study was conducted in the In-patient departments of General Medicine and Pulmonary medicine of Yenepoya Medical College Hospital. Considering the inclusion and exclusion criteria, patients were enrolled after 
taking written consent from each patient for the study. The study was carried out by considering the following criteria:

\section{Inclusion Criteria:}

Patients of either sex, aged 18 years and above from the InPatient departments of General Medicine and Pulmonary medicine. Can understand oral and written information Diagnosed with COPD or Asthma. Patients diagnosed with COPD based on GOLD guidelines

\section{Exclusion criteria:}

Patients admitted in Intensive Care Unit, other departments and out-patients. Patients with mental or psychiatric diseases, cognitive dysfunction. Patients on cancer chemotherapy and dialysis. Pregnant and lactating females.

\section{Source of Data:}

Patients' medical record, Patient interview, Data collection tools, Patient data collection form, Medication adherence scale (Morisky-8) ${ }^{3}, 36$ item short form health survey (SF-36 Questionnaire) 4, St. George's Respiratory Questionnaire (SGRQ) 5, Modified Medical Research Council (mMRC) Dyspnoea Scale, Patient Counselling documentation form

\section{Statistical Analysis:}

The collected data were tabulated and analyzed using Microsoft Excel version 13 and SPSS Version 22. The statistical analysis was done with McNemar's test for assessing adherence using MMAS-8 and Wilcoxon sign test was used for the assessment of quality of life using St. George's Respiratory Questionnaire and 36- Short Form Survey.

Ethical Clearance: The study was approved by the Yenepoya University Ethics Committee, Deralakatte, Mangalore and issued ethical clearance certificate.

\section{RESULTS AND DISCUSSION}

\section{Details of patients enrolled into the study:}

During the 6 months study period, total of 69 (Asthma \& COPD) patients were enrolled as per our inclusion and exclusion criteria. In this study, 7 (58.3\%) were males and 5 (41.7\%) were females with Asthma and 53 (93.0\%) males, 4 $(7.0 \%)$ were females with COPD and most of the patients were above 45 years old. About $10(83.3 \%)$ patients were with Asthma and 52 (91.2\%) were with COPD in this age group.

\section{Smoking status:}

Out of 69 subjects, 2 (16.7\%) were ex-smokers in Asthma, 8 $(66.7 \%)$ were non-smokers and $2(16.7 \%)$ were current smokers, whereas in COPD, 32 (56.1\%) were ex-smokers, 6 $(10.5 \%)$ were non-smokers and 19 (33.3\%) were current smokers.

\begin{tabular}{|c|c|c|c|c|}
\hline \multirow[t]{2}{*}{ Basic Variable } & \multicolumn{2}{|c|}{ Asthma } & \multicolumn{2}{|c|}{ COPD } \\
\hline & Male & Female & Male & Female \\
\hline $\begin{array}{l}\text { No. of patients } \\
\text { enrolled in the } \\
\text { study }\end{array}$ & $7(58.3 \%)$ & $5(41.7 \%)$ & $53(93.0 \%)$ & $4(7 \%)$ \\
\hline \multicolumn{5}{|l|}{ Age in years } \\
\hline $18-25$ & \multicolumn{2}{|c|}{$1(8.3 \%)$} & \multicolumn{2}{|r|}{$0(0.0 \%)$} \\
\hline $26-33$ & \multicolumn{2}{|c|}{$1(8.3 \%)$} & \multicolumn{2}{|r|}{$1(1.8 \%)$} \\
\hline $34-45$ & \multicolumn{2}{|c|}{$0(0.0 \%)$} & \multicolumn{2}{|r|}{$4(7.0 \%)$} \\
\hline$>45$ & \multicolumn{2}{|c|}{$10(83.3 \%)$} & \multicolumn{2}{|r|}{52 (91.2\%) } \\
\hline \multicolumn{5}{|l|}{ Smoking index } \\
\hline Ex-smokers & \multicolumn{2}{|c|}{$2(16.7 \%)$} & \multicolumn{2}{|r|}{$32(56.1 \%)$} \\
\hline Non- smokers & \multicolumn{2}{|c|}{$8(66.7 \%)$} & \multicolumn{2}{|r|}{$6(10.5 \%)$} \\
\hline Current smokers & \multicolumn{2}{|c|}{$2(16.7 \%)$} & & $19(33.3 \%)$ \\
\hline
\end{tabular}




\section{Assessment of Medication Adherence:}

Medication adherence was assessed using Morisky Medication Adherence Scale (MMAS-8). All the subjects of the study were provided with the questionnaire at the time of enrolment and subsequent follow-up was done after patient counselling for the assessment of medication adherence. The baseline data was compared with before and after counselling scores using Mc-Nemar's test.

Among all the enrolled patients in Asthma, 9 (75\%) were low adherent and 3 (25\%) showed medium adherence to the medication before counselling. After counselling, 4 (57.1\%) showed improvement from low to medium adherence and 3 $(42.9 \%)$ remained in the medium adherence category but this was not found to be statistically significant ( $p$ value $=0.125$ ).

In COPD, $43(75.4 \%)$ were low adherent to the medication and $14(24.6 \%)$ were medium adherent to the medication before counselling. After counselling, 20 (60.6\%) showed significant improvement from low to medium and 13 $(39.4 \%)$ remained medium adherent $(P$ value $=0.00)$.

\begin{tabular}{|c|c|c|c|c|c|c|c|}
\hline & \multirow{2}{*}{ DIAGNOSIS } & & & \multicolumn{2}{|c|}{ Scores-After } & \multirow{2}{*}{ Total } & \multirow{2}{*}{$p$ value } \\
\hline & & & & Low & Medium & & \\
\hline \multirow{12}{*}{ 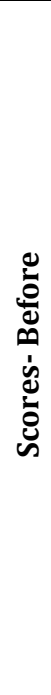 } & \multirow{6}{*}{ Asthma } & \multirow{2}{*}{ Low } & Count & 5 & 4 & 9 & \multirow{6}{*}{0.125} \\
\hline & & & Percent & $100.0 \%$ & $57.1 \%$ & $75.0 \%$ & \\
\hline & & \multirow{2}{*}{ Medium } & Count & 0 & 3 & 3 & \\
\hline & & & Percent & $0.0 \%$ & $42.9 \%$ & $25.0 \%$ & \\
\hline & & \multirow{2}{*}{ Total } & Count & 5 & 7 & 12 & \\
\hline & & & Percent & $100.0 \%$ & $100.0 \%$ & $100.0 \%$ & \\
\hline & \multirow{6}{*}{ COPD } & \multirow{2}{*}{ Low } & Count & 23 & 20 & 43 & \multirow{6}{*}{$0.00^{*}$} \\
\hline & & & Percent & $95.8 \%$ & $60.6 \%$ & $75.4 \%$ & \\
\hline & & Medium & Count & 1 & 13 & 14 & \\
\hline & & & Percent & $4.2 \%$ & $39.4 \%$ & $24.6 \%$ & \\
\hline & & \multirow{2}{*}{ Total } & Count & 24 & 33 & 57 & \\
\hline & & & Percent & $100.0 \%$ & $100.0 \%$ & $100.0 \%$ & \\
\hline
\end{tabular}

${ }^{*}$ statistically significant

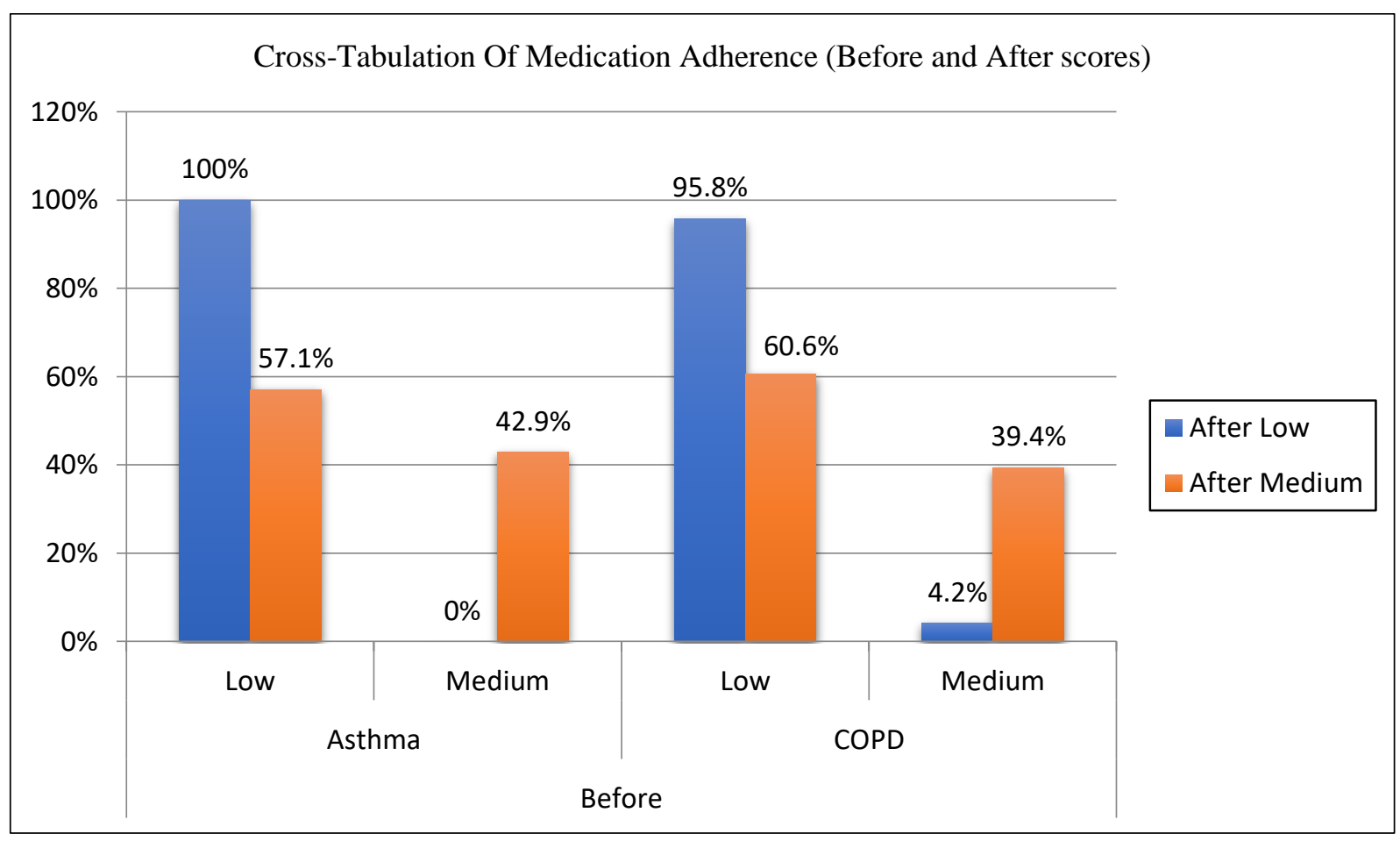

Figure 1: Cross-Tabulation of Medication Adherence (Before and after scores 


\section{Assessment of Quality of Life:}

Among the 69 subjects, $43 \%$ were known cases of COPD and $39 \%$ were known cases of Asthma. Newly diagnosed Asthma and COPD cases were of $9 \%$ each and more than $40 \%$ had one or more comorbidities and only $10 \%$ of patients were without any co- morbidities. Quality of life was assessed using St. George's Respiratory Questionnaire (SGRQ) and 36Short Form Survey Questionnaire (SF-36). All the subjects were provided with the above questionnaires at the time of enrolment, the follow up was done after patient counselling and the scores were compared using Wilcoxon sign test.

\section{St. George's Respiratory Questionnaire (SGRQ)}

In Asthmatic patients, the total SGRQ scores for the domain of symptom improved significantly after counselling (49.73 \pm 39.55 vs $58.44 \pm 39.81, \mathrm{p}<0.05$ ). Similarly, in COPD too, a significant improvement was observed in the total SGRQ scores for the domain of symptoms $(66.51 \pm 28.31$ vs $70.34 \pm$ 28.10, $\mathrm{p}<0.05$ ). Scores for Activities remained same in both Asthma $(93.7 \pm 10.87$ vs $93.7 \pm 11.5, \mathrm{p}>0.05)$ and COPD $(93.3 \pm 14.13$ vs $93.3 \pm 14.13, p<0.05)$ before and after counselling. Impact too showed significant improvement in the total SGRQ scores after counselling for both Asthma $(56.83 \pm 28.63$ vs $63.83 \pm 33.41, \mathrm{p}<0.05)$ and COPD $(56.8 \pm$ 28.58 vs $60.56 \pm 26.94, \mathrm{p}<0.05)$.

\begin{tabular}{|c|c|c|c|c|c|c|c|}
\hline \multicolumn{8}{|c|}{ Comparison of Symptoms } \\
\hline & & Subjects & Minimum & Maximum & Median & IQR & p value \\
\hline \multirow[t]{2}{*}{ Asthma } & Before & 12 & 19.97 & 93.04 & 49.73 & 39.55 & \multirow[t]{2}{*}{$0.002 *$} \\
\hline & After & 12 & 27.97 & 97.4 & 58.44 & 39.81 & \\
\hline \multirow[t]{2}{*}{ COPD } & Before & 57 & 33.04 & 95.03 & 66.51 & 28.31 & \multirow[t]{2}{*}{$0.00^{*}$} \\
\hline & After & 57 & 36.68 & 97.33 & 70.34 & 28.10 & \\
\hline \multicolumn{8}{|c|}{ Comparison of Activities } \\
\hline \multirow[t]{2}{*}{ Asthma } & Before & 12 & 42.52 & 100 & 93.7 & 10.87 & \multirow[t]{2}{*}{0.317} \\
\hline & After & 12 & 42.52 & 100 & 93.7 & 11.5 & \\
\hline \multirow[t]{2}{*}{ COPD } & Before & 57 & 54.43 & 100 & 93.3 & 14.13 & \multirow[t]{2}{*}{$0.022^{*}$} \\
\hline & After & 57 & 54.43 & 100 & 92.51 & 14.13 & \\
\hline \multicolumn{8}{|c|}{ Comparison of Impacts } \\
\hline \multirow[t]{2}{*}{ Asthma } & Before & 12 & 32.46 & 85.45 & 56.83 & 28.63 & \multirow[t]{2}{*}{$0.007^{*}$} \\
\hline & After & 12 & $33 . .46$ & 95.45 & 63.83 & 33.41 & \\
\hline \multirow[t]{2}{*}{ COPD } & Before & 57 & 35.84 & 87.4 & 56.8 & 28.58 & \multirow[t]{2}{*}{$0.00^{*}$} \\
\hline & After & 57 & 36.23 & 88.11 & 60.56 & 26.94 & \\
\hline
\end{tabular}
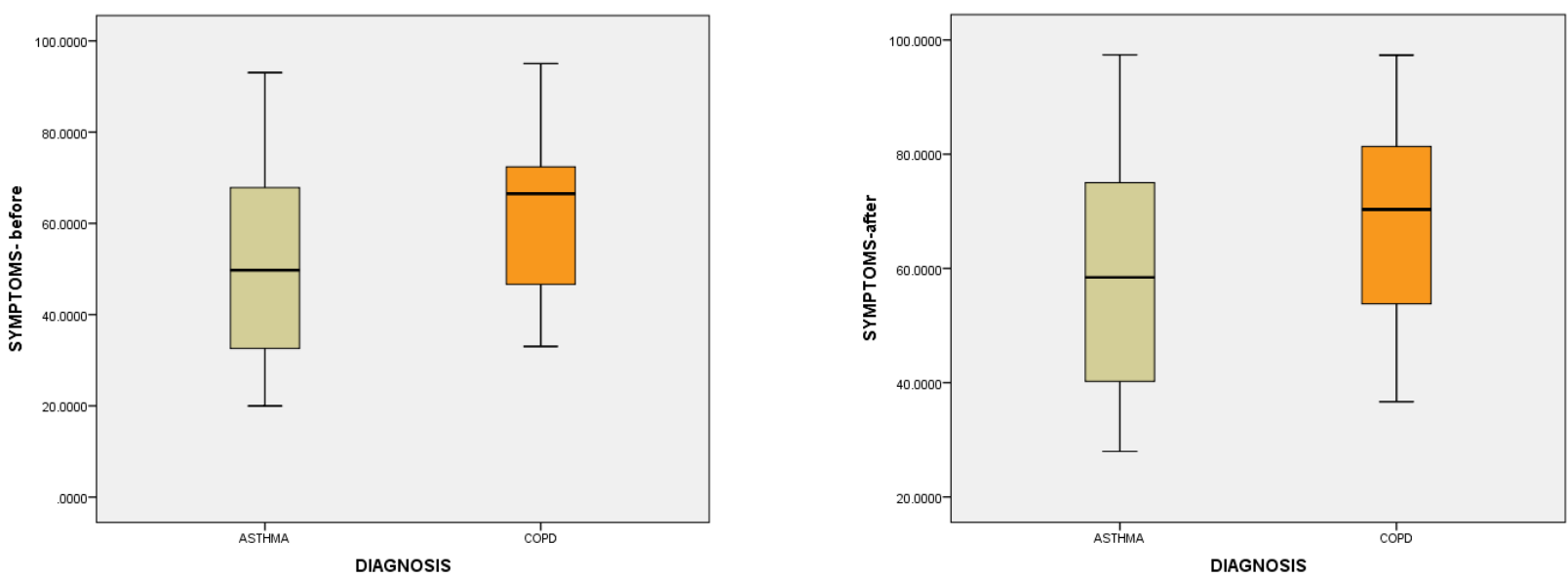

Figure 2(a): Comparison of symptoms before and after counselling 

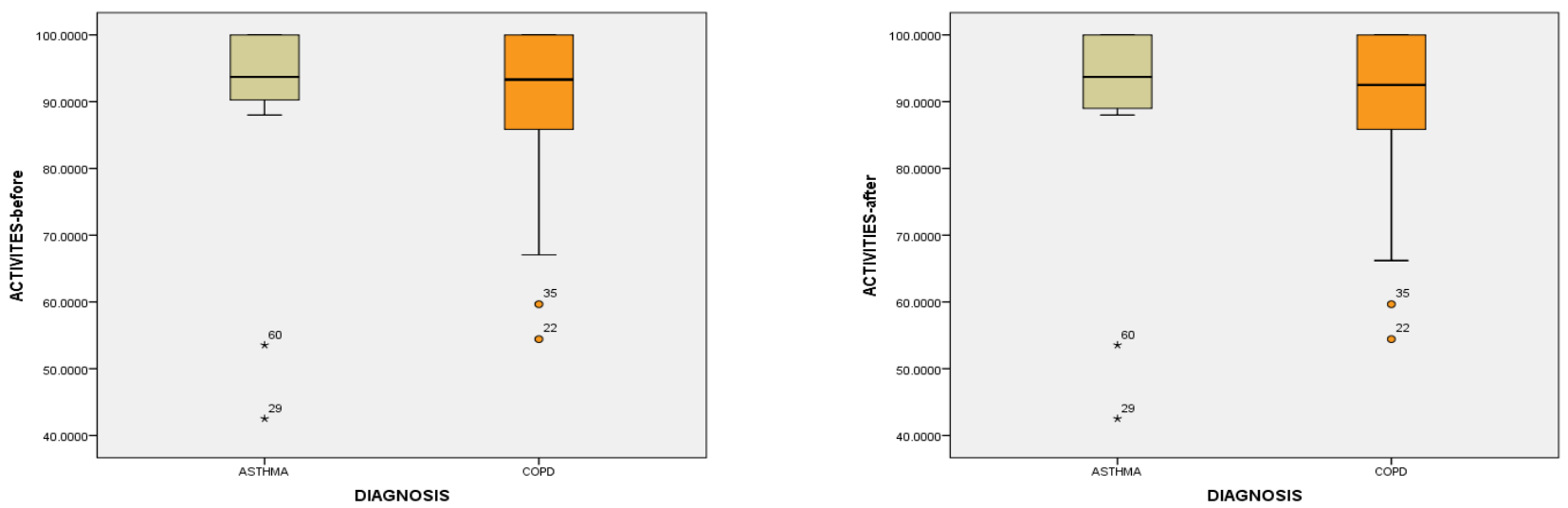

Figure 2(b): Comparison of activities before and after counselling
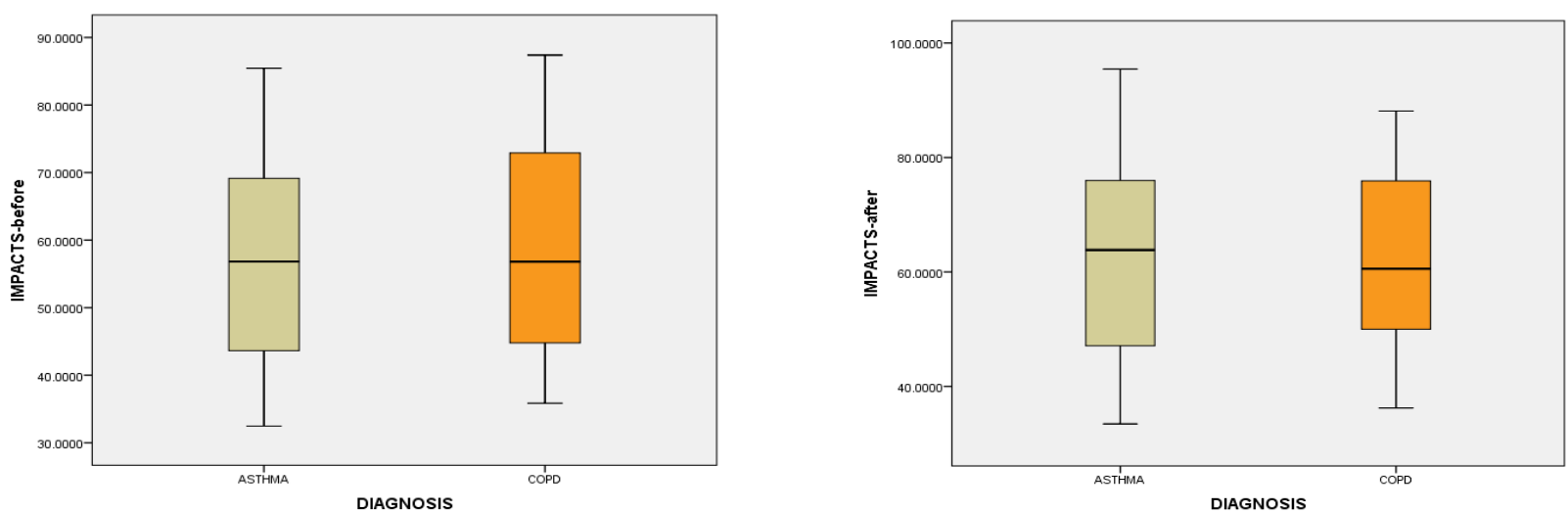

Figure 2(c): Comparison of impacts before and after counselling

\section{6-Short Form Survey (SF-36):}

For Asthma and COPD patients, SF-36 was used to assess quality of life before and after counselling and an average score was taken for assessment of significance value. Asthma patients showed significant improvement in quality of life from a median of 318.05 to a median of $674(p<0.05)$. Similarly, in COPD too there was a significant improvement in the quality of life from a median of 377.5 to a median of $754(\mathrm{p}<0.05)$.

\begin{tabular}{|c|c|c|c|c|c|c|c|}
\hline & & & & & & & \\
\hline & & & & & & & \multirow{3}{*}{$0.002^{*}$} \\
\hline \multirow{2}{*}{ Asthma } & Before & 12 & 184.5 & 481 & 318.05 & 223.1 & \\
\hline & After & 12 & 364 & 948 & 674 & 448.87 & \\
\hline \multirow{2}{*}{ COPD } & Before & 57 & 145.5 & 595 & 377.5 & 199.9 & \multirow{2}{*}{$0.00^{*}$} \\
\hline & After & 57 & 300 & 1163 & 754 & 385.45 & \\
\hline
\end{tabular}
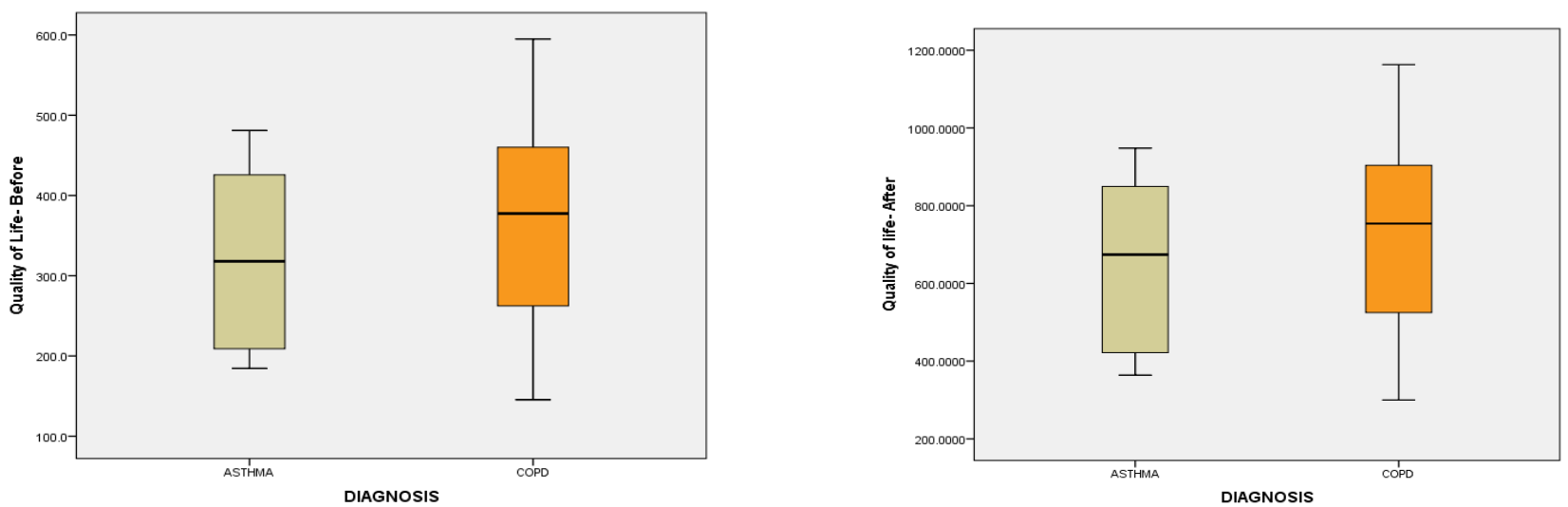

Figure 3: Comparison of Quality of Life before and after patient counselling using SF-36 


\section{DISCUSSION}

Asthma and COPD pose a large health burden across health systems and consistently rank among the most fatal diseases across developed countries. The study was designed to assess the role of clinical pharmacist in enhancement of medication adherence and quality of life in Asthma and COPD patients.

The study was conducted using various questionnaires to assess the medication adherence and quality of life. Individualized patient counselling was carried out regarding the disease, medications and lifestyle. 6-8

Significant improvement in compliances to the medications was observed by patient counselling. This result shows the role of clinical pharmacist in the enhancement of medication adherence in Asthma and COPD patients. The major selfreported reasons for non-adherence to medication are symptomatic improvement, forgetfulness, expense, illiteracy, fear of side effects, feel non-beneficial. The same reasons were reported in the previous studies, hence this study focused on these reasons to minimize by clinical pharmacistmediated patient counselling. ${ }^{9}$

The current study also addressed the improvement of quality of life by clinical pharmacist-mediated patient counselling. SGRQ shows improvement in the domains of symptoms and impacts and SF-36 showed significant improvement in all the domains. The participating clinical pharmacists acquired new communication skills in their dealings with patients. Good communication reflects patient education and tailoring therapy for patients' better health related outcome. 10-11

\section{CONCLUSION}

The study revealed that the enhancement of medication adherence and quality of life was statistically significant in Asthma and COPD patients after clinical pharmacist mediated patient- counselling. The self-reporting questionnaire method was found to be effective for the assessment of medication adherence and quality of life. The reasons for non-adherence to the medication were resolved by patient counselling and hence, quality of life was improved which resulted in better patient health-related outcome. Clinical pharmacists must be considered as an integral element of healthcare system.

\section{ACKNOWLEDGEMENT}

We wish to acknowledge the Principal, HOD and all staffs of Shree Devi College of Pharmacy, Mangalore. We sincerely thank our guide Dr Cyril Tom, Assistant Professor, Shree Devi College of Pharmacy, Mangalore and our co-guide $\mathrm{Dr}$ Prabhakar Adake, Associate Professor, Department of Pharmacology, Yenepoya Medical College Hospital for being our constant source of inspiration and encouragement for carrying out this project. We also thank all the staffs from the Department of General Medicine Yenepoya Medical College Hospital, Derlakatte, Mangalore for guiding us in the successful completion of this project.

\section{REFERENCES}

1. Fugate AR, Kadam AM, Ganachari MS. Prospective Study of Medication Adherence Pattern in Chronic Obstructive Pulmonary Disease and Asthma Patient's in Tertiary Care teaching Hospital. Indian Journal of Pharmacy Practice. 2015 Apr-June; 8(2):78-83. https://doi.org/10.5530/ijopp.8.2.6

2. Emilija N, Aleksander N, Ruza S, Tijana B, Veselin M, Jelena M. Model for Developing a Health-Related Quality of Life Questionnaire for Chronic Obstructive Pulmonary Disease. Canadian Respiratory Journal. 2018 May:1-6. https://doi.org/10.1155/2018/6450962

3. Morisky DE, Green LW, Levine DW. Concurrent and predictive validity of a self- reported measure of medication adherence. Medical care 1986; 24:67-74

https://doi.org/10.1097/00005650-198601000-00007

4. Hays, R.D, Sherbourne et.al., The RAND 36 item Health survey 1.0. Health economics, 1993; 2:217-227 https://doi.org/10.1002/hec.4730020305

5. Jones PW, Quirk FH, BaveystockCM.The St. George's Respiratory Questionnaire. Resp Med 1991; 85(suppl B):2531. https://doi.org/10.1016/S0954-6111(06)80166-6

6. Takemura M, Mitsui K, Itotani R, Ishitoko M, Suzuki S, Matsumoto $M$ et al.Relationship between repeated instruction on inhalation therapy, medication adherence and health status in COPD. Interntional Journal of COPD. 2011 January: 6 https://doi.org/10.2147/COPD.S16173

7. Arief M, Satyanarayana B, Wajid S, Paladugu ND, Pasha SI, Poloju D et al.Clinical pharmacist role in the management of Asthma in tertiary care hospital. Journal of Current Chemical and Pharmaceutical Sciences. 2013; 3(2):100-112.

8. Gillissen A. Patients' adherence in Asthma. Journal of Physiology and Pharmacology. 2007; 58(5):205-222.

9. Xin C, xia z, jiang C, lin M, li G. The impact of pharmacist- managed clinic on medication adherence and health-related quality of life in patients with COPD: a randomized controlled study. patient preference and adherence. 2016; 10:1197-1203 https://doi.org/10.2147/PPA.S110167

10. Apikoglu-Rabus S, Yesilyaprak G, Izzettin FV. Drug-related problems and pharmacist interventions in a cohort of patients with asthma and chronic obstructive pulmonary disease. Respiratory medicine. 2016; 120:109-115. https://doi.org/10.1016/j.rmed.2016.10.006

11. Mallesh M, Purushothama Reddy K, P.Vijaya Narasimha Reddy. Evaluation of the Clinical Pharmacist Role in a Health Care Team; a Comarative Approch. Indian journal of pharmacy practice. 2016; 9(4):236-46.

https://doi.org/10.5530/ijopp.9.4.5 